\title{
Numerical Investigation of Secondary Flows in a High-lift Low Pressure Turbine
}

\author{
J. Cui ${ }^{\mathrm{a}, *}$, V. Nagabhushana Rao ${ }^{\mathrm{a}}$, P. G. Tucker ${ }^{\mathrm{a}}$ \\ ${ }^{a}$ CFD laboratory, Department of Engineering, University of Cambridge
}

\begin{abstract}
In turbomachines, secondary flows (or endwall flows) typically originate at the junction between endwalls and the blade surface. Within the blade passage, the strength of the secondary flows is amplified by the crossflow from the pressure to the suction surface of the blade. The enhanced mixing due to secondary flows induce additional losses into the system. This decreases the overall work output and also changes the flow incidence onto the downstream blade rows. Using a series of high-fidelity eddy resolving simulations, the current study attempts to provide an improved understanding for the complex flow physics over the endwalls of a high-lift Low Pressure Turbine (LPT) blade. The effect of three different inflow conditions has been studied. These include a laminar boundary layer (LBL), a turbulent boundary layer (TBL) and wakes with secondary flow (W\&S) from an upstream blade row. For the simulations with TBL and W\&S, precursor eddy resolving simulations were used to prescribe realistic inflows. The loss generation mechanisms were subsequently studied both at the endwall and the midspan, which includes evaluating the mass-averaged total pressure loss coefficient $\left(Y_{p}\right)$ and the loss generation rate.

When compared to LBL, additional disturbances from an incoming TBL and wakes with secondary flows enhanced the mixing within the blade passage resulting in a substantial increase in the total pressure loss. Prior to flow transition, incoming wakes with secondary flows increased the local loss generation rate at both the endwall and the midspan in the front portion of the blade passage $\left(x / C_{x}<0.84\right)$. In contrast, in the
\end{abstract}

\footnotetext{
* Corresponding author

Email address: jc763@cam.ac.uk (J. Cui)
} 
aft portion of the passage $\left(x / C_{x}>0.8\right)$, the incoming wakes effectively suppressed the separation bubble at the midspan thereby decreasing the local loss generation rate. It is also demonstrated that the wakes shed from the trailing edge at the mid-span mix out rapidly when compared to the passage vortex at the endwall.

Keywords: low pressure turbine, endwall flow, loss mechanism, CFD

\section{Introduction}

The efficiency of a low pressure turbine (LPT) strongly influences the overall performance of a modern gas turbine aero-engine. The LPT also contributes to about $30 \%$ of the total weight of the engine [1]. High-lift blade designs are used in the modern aero

5 engines. Such designs can extract the same amount of work output with a lower blade count thereby reducing the weight of the engine. However, high-lift blades increase a) the risk of boundary layer separation on the suction surface and b) the strength of secondary flows. There are several studies addressing the issue of the midspan separation and its control [2, 3, 4]. The current authors [5] have also reported the effect of different external disturbances on the separated shear layer transition at the midspan.

Secondary flows typically generate around $30 \%$ of the overall loss in a blade row. Most of the previous investigations of secondary flows have either been experimental or low-fidelity numerical studies (using Reynolds Averaged Navier-Stokes - RANS). The objectives of these studies can be broadly classified into understanding the: a) endwall flow features, b) loss generation mechanisms and c) effect of inflow conditions. A brief review of the relevant literature is given below:

Flow features at endwall: Early studies on secondary flows focused on understanding the overall flow features [6]. Figure 1 ] shows the widely accepted topology of the endwall flow. The incoming boundary layer separates under adverse pressure gradient, which forms the suction and pressure legs of the horseshoe vortex. Subsequently, the pressure leg merges with the suction leg to form the passage vortex. However, disagreement still exists regarding the details of the secondary flow structure. One of them is the development of the suction leg of the horseshoe vortex. Sharma and Bulter [7] and Wang et al. [8] demonstrated that this leg wrapped around the passage vortex, 
eventually merging with the passage vortex. On the other hand, Goldstein and Spores [9] argued that the suction side leg of horseshoe vortex always travelled above the passage vortex. The origin of the counter vortex downstream of the trailing edge is also an open question. Hodson and Dominy [10] proposed that the relative skew of the boundary layer over the blade results in a counter vortex, while Wang et al. [8] showed that the counter vortex is induced by the strong passage vortex.

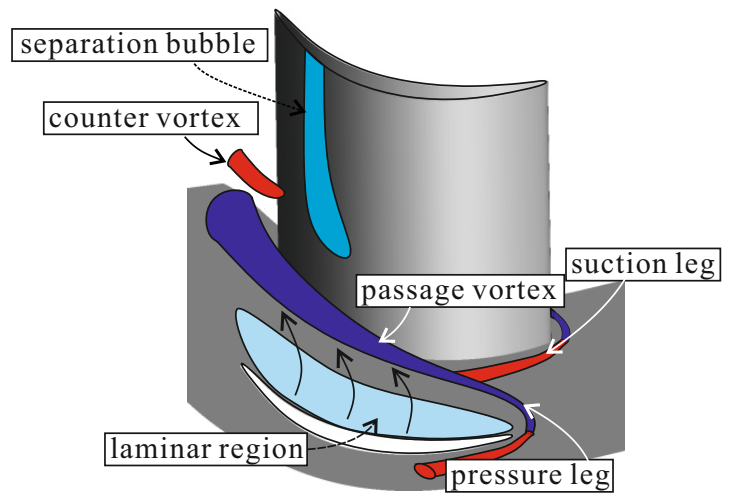

Figure 1: Sketch of typical endwall flow structures in low pressure turbines.

Loss generation mechanisms: Secondary kinetic energy is often used to quantify the total endwall loss. However, the lack of correlation between secondary kinetic energy and total loss is reported by Denton and Pullan [11]. Ingram et al. [12] also demonstrated that a blade designed with a reduced level of secondary kinetic energy can still increase loss. The relation between loss generation rate and Reynolds stresses has been experimentally investigated by Macisaac et al. [13]. The importance of shear stress in the loss generation downstream of the trailing edge was also demonstrated by these workers. Due to the limitation of accessibility within the blade passage, only measurements downstream of the trailing edge were reported in [13].

Effect of inflow conditions: Blanco et al. [14] reported that the state of the incoming endwall boundary layer plays an important role in endwall loss generation. By simulating the wakes using upstream moving bars, Steurer [15] studied the effects of incoming wakes on the secondary flow. The effect of secondary flow from the upstream blade row was not considered in this study. 
The investigations summarized above have provided valuable insights into the physics of secondary flows. However, the knowledge of flow physics and loss generation mechanisms concerning endwall flows is still limited; either due to the restricted access to the endwall zones in measurements (and general flow complexity) or due to the assumptions involved in the low-fidelity models. This lack of understanding impedes the development of more accurate secondary loss prediction models and the flow control methods for loss reduction. Using a series of high-fidelity eddy resolving simulations, the current study attempts to provide an improved understanding of the endwall flows and the associated loss generation mechanisms in high-lift low pressure turbines. The effect of three different inflow conditions have been studied. These include a laminar boundary layer (LBL), a turbulent boundary layer (TBL) and wakes with secondary flow (W\&S) from an upstream blade row.

This paper is organized as follows. Section 2 briefly describes the numerical methodology and computational domain used in the current study. Section 3 presents a detailed investigation of the endwall flow features and loss mechanisms both with and without the influence of different inflow conditions. Section 4 concludes the study.

\section{Computational Methodology}

The endwall of a T106A cascade has been considered in the current study. The T106A cascade has been widely used in the research community in the context of studying transitional flow over the suction surface at midspans. Table 1 gives the key design parameters of the T106A cascade. Figure 2 (a) shows the computational domain and boundary conditions in the $x-y$ plane. This domain was extruded in $z$ direction to resolve half a blade span $(50 \% h)$. No-slip and inviscid wall boundary conditions were imposed on the bottom and top boundaries, respectively. The latter assumption and its implications are described further later.

\subsection{Inflow boundary conditions}

In total, three simulations with different inflow conditions have been carried out. An overview of the test cases is given in Table 2. The mean velocity profiles at the 
Table 1: Specification of T106A cascade

\begin{tabular}{ll}
\hline & \\
Reynolds number $(R e)$ & $1.6 \times 10^{5}$ \\
Chord $(C)$ & $198 \mathrm{~mm}$ \\
Axial chord $\left(C_{x}\right)$ & $170 \mathrm{~mm}$ \\
Pitch & $158 \mathrm{~mm}$ \\
Span $(h)$ & $375 \mathrm{~mm}$ \\
Inlet angle $\left(\alpha_{1}\right)$ & $-39.7^{\circ}$ \\
Design exit flow angle $\left(\alpha_{2}\right)$ & $63.2^{\circ}$ \\
Flow coefficient $(\Phi)$ & 0.83 \\
Inlet velocity $\left(U_{i n}\right)$ & $\approx 6.8 \mathrm{~m} / \mathrm{s}$ \\
Outlet velocity $\left(U_{T E}\right)$ & $\approx 12 \mathrm{~m} / \mathrm{s}$ \\
\hline
\end{tabular}

inlet were specified based on the measurements of Blanco [16]. Figure 3 shows the incoming mean velocity and the associated turbulent kinetic energy profiles imposed at the inlet for the LBL and TBL test cases, respectively. For the TBL test case, a precursor direct numerical simulation was carried out using the Lund's recycling technique [17] to generate the time series data of the streamwise growing turbulent boundary layer. The time-series data was saved at an axial location where $R e_{\theta} \approx 1000$. This time-series data was subsequently fed onto the inflow boundary.

For the test case with incoming wakes and secondary flow (W\&S), the time-series of velocity components was extracted from the LBL case, downstream of the trailing edge at $x / C_{x}=1.01$. The velocity magnitude was scaled down to match the Reynolds number of the LBL and TBL cases. The method of velocity triangles [18] was used to ensure that the pressure distribution at the midspan remains unaltered. The upstreamrotor velocity was set to $0.96 \% U_{i n}$. This satisfies the condition of a repeating stage at $50 \%$ reaction. Figure 2(b) shows a snapshot of vorticity magnitude contours at the inflow and also at the endwall. As can be seen, the passage vortex and the wake from the upstream rotor have been replicated using this method. 

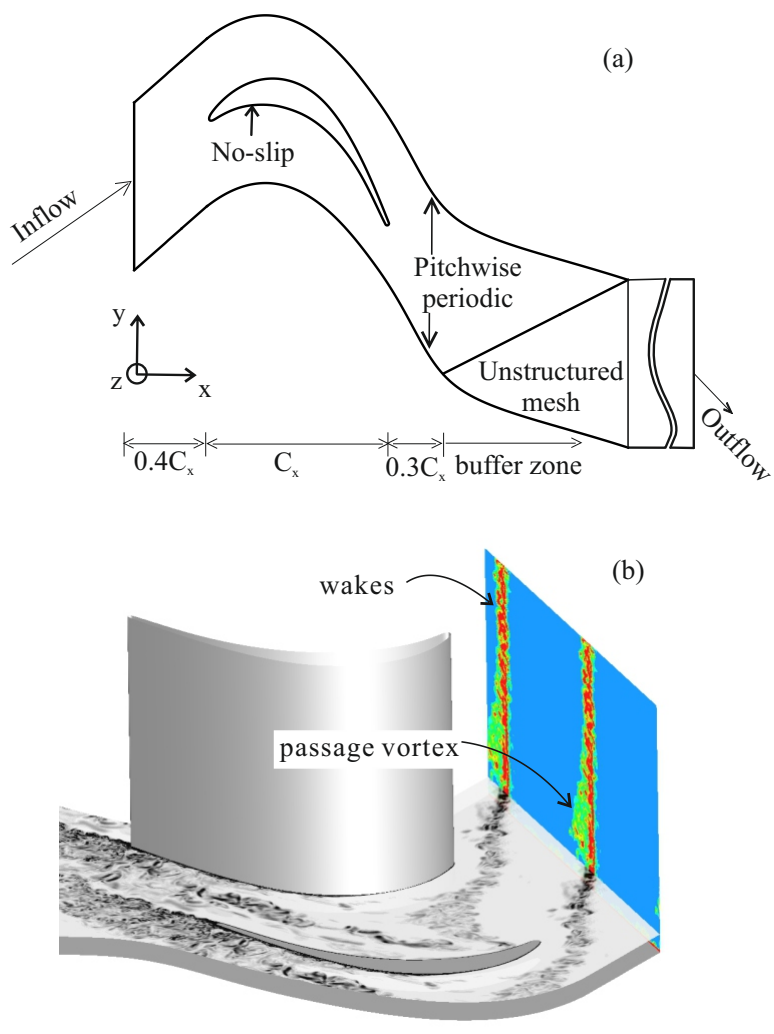

Figure 2: Domain, boundary conditions and inflow: (a) computational domain and boundary conditions and (b) vorticity magnitude on the inflow and endwall for the W\&S case.

\subsection{Solver characteristics}

Simulations were carried out using the Rolls-Royce's in-house solver - HYDRA [19], which has been extensively validated for a variety of transitional and turbulent flows [5, 20]. The solver is second order accurate in both space and time. It is an unstructured, edge-based finite volume code. The solver also utilizes artificial compressibility approach of Rogers et al. [21] for an improved computational performance

95 at low-Mach numbers. For all the cases investigated, the flow has been time-averaged for 10 through flows based on the chord length $(C)$ and the incoming velocity $\left(U_{i n}\right)$. 
Table 2: Test cases.

\begin{tabular}{ll}
\hline Test cases & Inflow condition \\
\hline LBL & laminar boundary layer \\
TBL & turbulent boundary layer \\
W\&S & incoming wakes with secondary flow
\end{tabular}
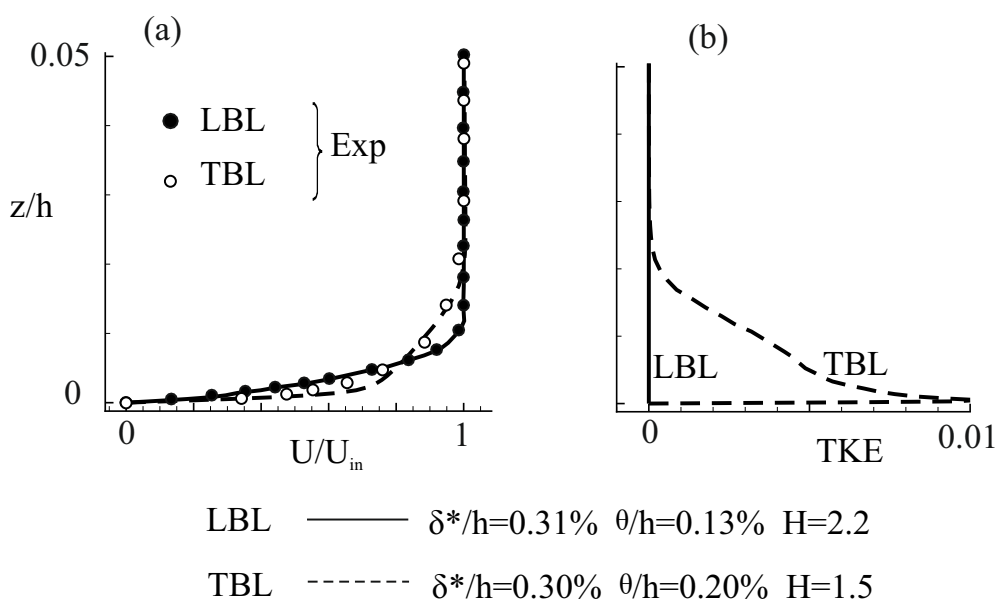

Figure 3: Inflow profiles for the LBL and TBL cases: (a) velocity profiles and (b) turbulent kinetic energy profiles

\subsection{Grid resolution}

Around 40 million cells were used to simulate each of the cases given in Table 2. The grid spacings (in wall units) in the region of primary interest (i.e. on the endwall and suction surface) are within the range of $\Delta y^{+}<2, \Delta x^{+}<40$ and $\Delta z^{+}<50$. The mesh resolution complies with the recommended values for the wall-resolved LES. Grid independence study has been carried out for the TBL test case. The mesh was refined by 1.5 times in spanwise and 1.4 times in pitchwise direction, while the number 
of points in the streamwise direction was left unaltered. This results in the final refined mesh with around 85 million cell count.

\section{Results and Discussion}

\subsection{Grid independence study}

Figure 4(a) compares the axial evolution of the stagnation pressure loss coefficient $\left(Y_{p}=\left(P_{01}-P\right) /\left(P_{01}-P_{2}\right) \sqrt{1}\right.$ for both the baseline and refined meshes. Here, $P$ is the plateau of pressure coefficient between $x / C_{x}=0.8-0.92$ on the suction surface indicates a closed separation bubble. The shape factor increases to around 3.5 in the separated region (see figure 5(b)). The separated shear layer transitions to turbulence

\footnotetext{
${ }^{1}$ The stagnation pressure loss in figure 4 a) has been mass averaged in the pitchwise direction using equation 2
} 

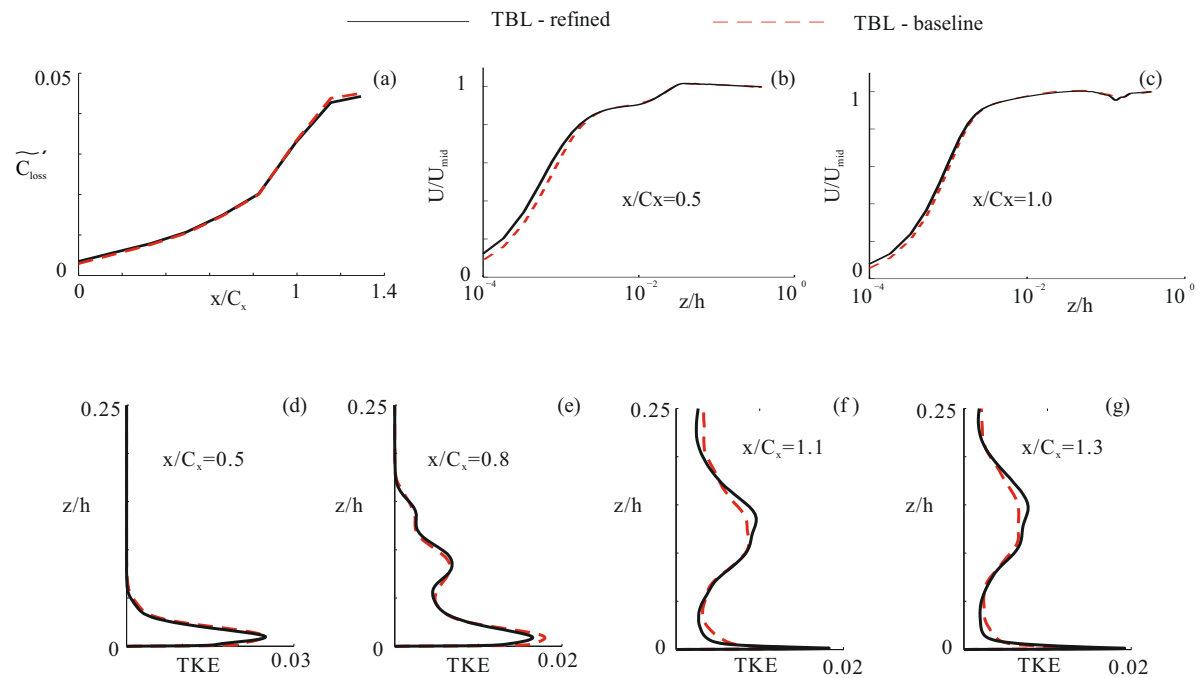

Figure 4: Comparison between simulations with the baseline (- - - - ) and refined (_ $-(-)$ meshes for (a) total pressure loss coefficient, (b,c) pitchwise averaged mean flow velocity magnitude profiles, and (d-g) pitchwise averaged turbulent kinetic energy profiles.

at around $x / C_{x}=0.92$ which drives the flow reattachment. The shape factor after reattachment is around 1.6, which is typical of a turbulent boundary layer.

\subsection{Overall flow features on the endwall}

The key features of the endwall flow are illustrated in figure 6 using the iso-surfaces of $\left.\mathrm{Q}\right|^{2}$ contoured with $U / U_{i n}$. Frames (a) and (b) show the instantaneous and timeaveraged flow fields, respectively. It is evident that, as the flow approaches the leading edge of the blade, the boundary layer at the endwall separates due to the adverse pressure gradient. The boundary layer rolls up to form a horseshoe vortex. This vortex subsequently splits into the pressure and suction legs which convect into different blade passages. The pressure leg combines with the cross-flow from the pressure to suction surface thereby forming a passage vortex - a dominant flow structure in secondary flows. Consistent with the observations of Goldstein and Spores [9], the vortex

\footnotetext{
${ }^{2} Q=1 / 2\left(|\boldsymbol{\Omega}|^{2}-|\mathbf{S}|^{2}\right)$, where $\boldsymbol{\Omega}$ is the vorticity tensor and $\mathbf{S}$ is the rate-of-strain tensor
} 

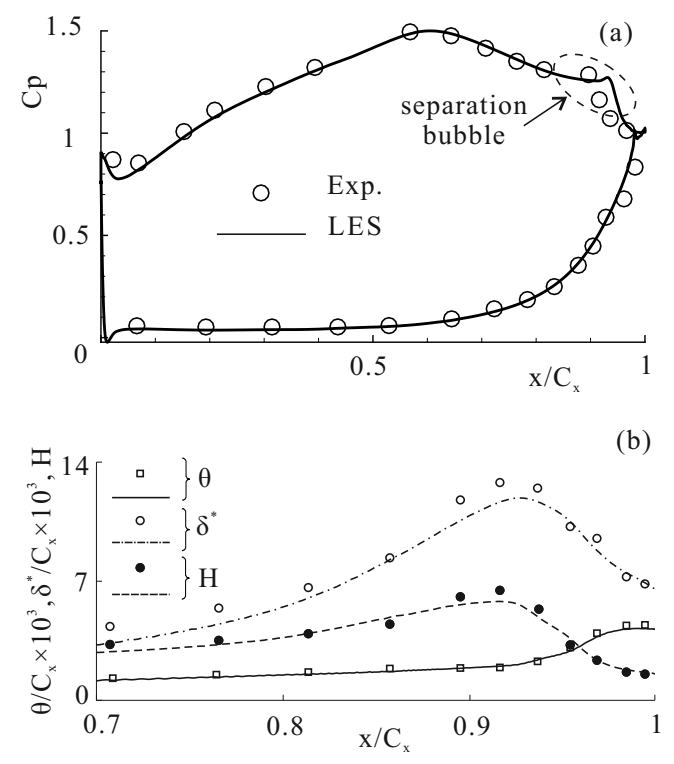

Figure 5: Comparisons with measurements at midspan: (a) pressure coefficient distribution and (b) boundary layer integral parameters on the suction surface. Symbols: measurements by Opoka [3] and lines: numerical results. $\delta^{*}$ - boundary layer displacement thickness, $\theta$ - boundary layer momentum thickness, $H$ - shape factor

travelling above the passage vortex, labelled as vor ${ }_{A}$, eventually merges with the passage vortex at the trailing edge. This vortex has a different sense of rotation relative to the passage vortex.

A new boundary layer is formed downstream of the pressure leg. This new boundary layer is largely laminar and independent of the state of the incoming endwall boundary layer. Figure 7 demonstrates this more clearly using the contours of shape factor ${ }^{3}$ close to the endwall for the TBL case. Recall that the shape factor of a laminar Blasius boundary layer is around 2.5 , while the shape factor of a turbulent boundary layer is around $1.3-1.4$. Notice that the zone downstream of the pressure leg is largely laminar (with a shape factor of around 2.5) even with an incoming turbulent boundary layer (where the shape factor is around 1.5). This is expected because most of the incoming boundary layer is separated and swept away from the endwall and the pressure surface.

\footnotetext{
${ }^{3}$ Note that shape factor is not evaluated in the highly three-dimensional region
} 


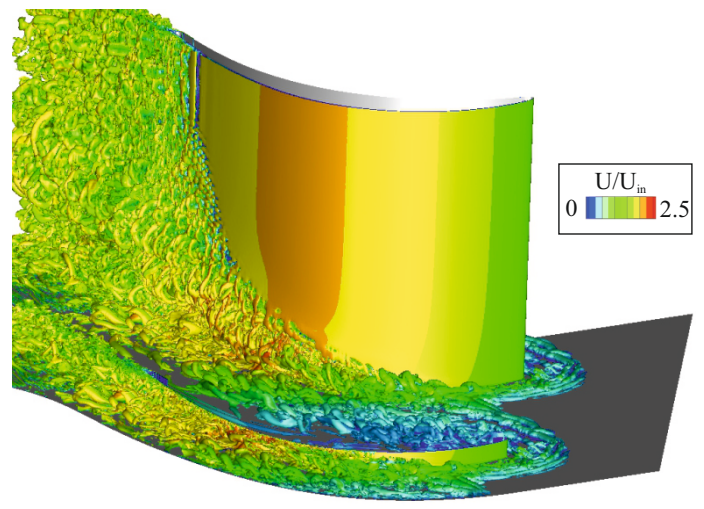

(a)

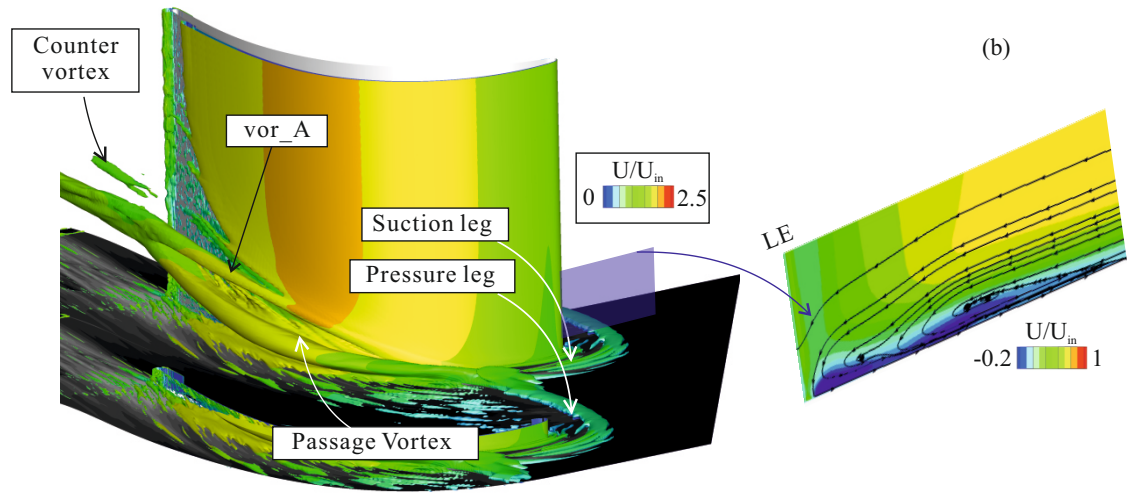

Figure 6: Q-criterion isosurface coloured by velocity magnitude for case LBL: (a) instantaneous and (b) time-averaged flow solutions.

This laminar boundary layer can even extend to $30 \% C_{x}$ downstream of the trailing edge.

The general flow structure for the TBL and W\&S cases is identical to the incoming LBL case except that the pressure leg of the horseshoe vortex is smaller and it moves axially downstream. This is due to the fact that the additional disturbances in TBL and $\mathrm{W} \& \mathrm{~S}$ cases aid the flow in overcoming the adverse pressure gradient when compared to the LBL case. 


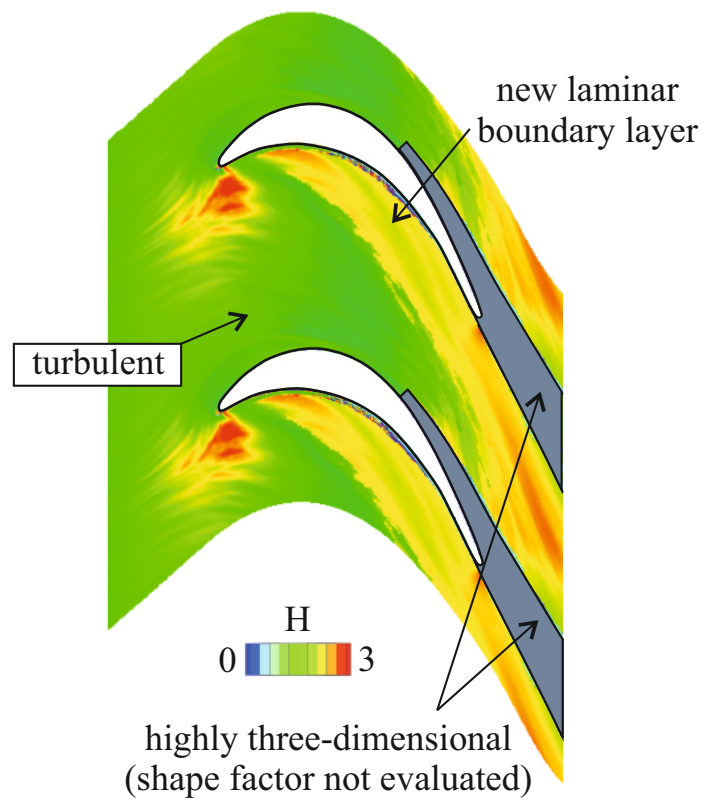

Figure 7: Shape factor on the endwall of TBL case.

160

\subsection{Loss analysis for LBL case}

In this section, a loss analysis will be introduced and carried out for the LBL test case. Additionally, the effects of different inflow boundary conditions will be addressed in the subsequent section 3.5 . The entire loss analysis (evaluation of turbulent kinetic energy, total loss generation rate, etc) in the upcoming sections has been estimated over the axial planes shown in figure 8 (a). In this analysis, turbulent kinetic energy and loss generation rate are area averaged using:

$$
\text { Area average: } \quad \dot{\widetilde{\phi}}=\frac{1}{A} \int_{0}^{0.5 h} \int_{0}^{P_{y}} \phi d y d z
$$

on the other hand the mass averaged total pressure loss coefficient is estimated using

$$
\text { Mass average: } \quad \widetilde{\phi}^{\prime}=\frac{\rho}{\dot{m}} \int_{0}^{0.5 h} \int_{0}^{P_{y}} \phi u d y d z
$$

Here, $\phi$ is the quantity of interest. Also, $P_{y}$ and $h$ are the pitch and span of the blade, $A$ and $\dot{m}$ are the cross-sectional area of the blade passage and the corresponding mass flow rate through it, respectively. 
To further facilitate the analysis, the resolved span is categorized into two dimensional (2D) and three dimensional (3D) regions as shown in figure $8 \mathrm{~b}$ ). The 3D region $(z / h<25 \%)$ is effected by the three-dimensional endwall flow. The flow in the 2D region over rest of the span $(z / h=25 \%-50 \%)$ is quasi two-dimensional where the boundary layer separates on the suction surface and transitions to turbulence.

\subsubsection{Loss cores}

The cumulative loss generated within the blade passage can be quantified using the mass-averaged stagnation pressure loss coefficient $Y_{p}$ downstream of the trailing edge. Figure $8 \mathrm{~b}$ ) shows the isolines of $Y_{p}$ at $x / C_{x}=1.3$ contoured with streamwise vorticity. A typical three loss-core system has been captured. These cores, denoted as I, II and III, correspond to the stagnation pressure loss due to the counter, passage and corner vortices, respectively. From the streamwise vorticity contours in figure 8 (b), it is evident that the passage and counter vortices rotate in opposite direction to each other. The counter rotating motion convects low momentum fluid from the wake into the main stream, and thus increases mixing. The enhanced mixing largely contributes to the loss generation associated with the endwall flow downstream of the trailing edge. More discussion on this will be given in section 3.4.3.

\subsubsection{Turbulent kinetic energy and loss coefficient within blade passage}

Figure $9(a, b)$ show the axial evolution of the area-averaged turbulent kinetic energy (TKE) and mass-averaged total pressure loss coefficient within the blade passage. On each axial plane extracted, the 2D and 3D categorization introduced in figure $8 \mathrm{~b}$ ) is used to examine the loss contribution from $2 \mathrm{D}$ and $3 \mathrm{D}$ regions.

Figure 9 (a) shows that the TKE in the 3D region immediately increases downstream of the leading edge due to the development of the three dimensional separation on the endwall. The increase in TKE is gradual upto $x / C_{x}=0.64$ where the pressure gradient is favourable. However, it significantly increases in the region of adverse pressure gradient between $x / C_{x}=0.64-1.0$. This is due to the development of large passage vortex. Beyond $x / C_{x}=1.0$, the TKE decreases gradually, by around $25 \%$ between the tailing edge and the last extraction plane $\left(x / C_{x}=1.3\right)$. This is due to the 


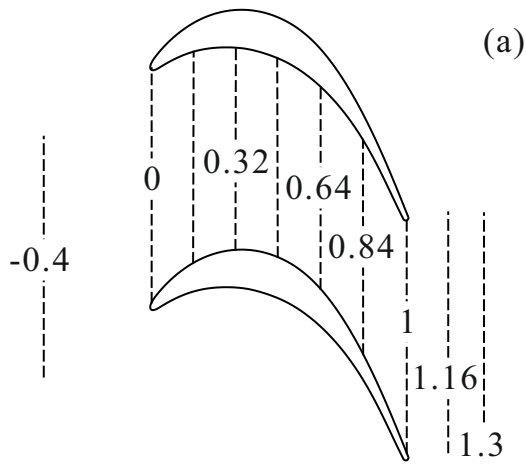

(a)

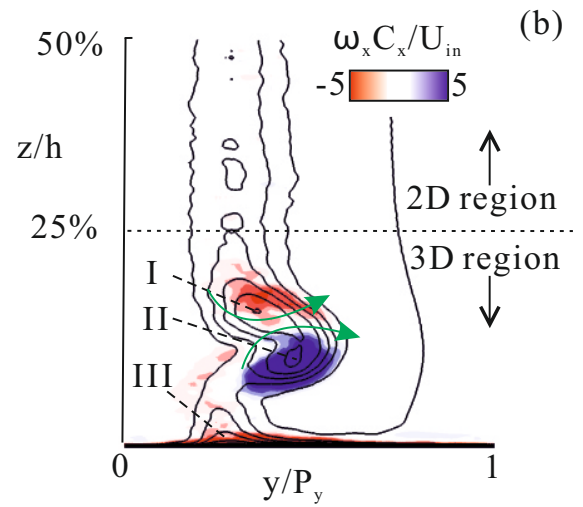

Figure 8: (a) Axial locations of extracted planes; (b) isolines of local stagnation pressure loss coefficient superimposed with streamwise vorticity at $x / C_{x}=1.3$.

absence of blade wetted area. On the other hand, within the 2D region, the boundary layer is largely laminar upto $x / C_{x}=0.84^{4}$ Beyond this point, the flow transitions and a dramatic increase in TKE is evident. At $x / C_{x}=1.0$, the contribution from both $2 \mathrm{D}$ and $3 \mathrm{D}$ regions to the overall TKE is almost identical. However, downstream of the trailing edge $\left(x / C_{x}=1.0-1.3\right)$, it is evident that the decrease in TKE within the $2 \mathrm{D}$ region is much steeper than in the $3 \mathrm{D}$ region. It suggests that the wake from the trailing edge diffuses faster when compared to the passage vortex. This aspect will be revisited in the subsequent section 3.4 .3 on loss mechanisms.

\footnotetext{
${ }^{4}$ Note the actual transition point is at $x / C_{x}=0.92$. Since there is no extracting plane between $x / C_{x}=$ $0.84-1.0$, the transition point is identified at $x / C_{x}=0.84$ in the analysis based on the extracting planes shown in figure 8 a)
} 
Figure 9(b) shows the mass averaged total pressure loss coefficient within the passage. The overall loss is again categorized into the loss accumulated within the $2 \mathrm{D}$ and

\subsubsection{Loss mechanisms}

The mean flow kinetic energy equation is used to study the loss generation rate within the flow passage. This can be written as

$$
\frac{\partial E}{\partial t}+\bar{u}_{j} \frac{\partial E}{\partial x_{j}}=\underbrace{\frac{\partial}{\partial x_{j}}\left(-\bar{u}_{j} \frac{\bar{p}}{\rho}+2 \nu \bar{u}_{i} \bar{s}_{i j}-\overline{u_{i}^{\prime} u_{j}^{\prime}} \bar{u}_{i}\right)}_{\text {I }} \underbrace{-2 \nu \bar{s}_{i j} \bar{s}_{i j}}_{\text {II }} \underbrace{+\bar{u}_{i}^{\prime} u_{j}^{\prime}}_{\text {III }} \bar{s}_{i j}
$$

where $E=\frac{1}{2} \bar{u}_{i} \bar{u}_{i}$ is the mean flow kinetic energy, $s_{i j}$ is the strain tensor and $\overline{u_{i}^{\prime} u_{j}^{\prime}}$ is the time averaged Reynolds stress. The first term (I) on the right hand side of equation 3 transports/redistributes the mean-flow kinetic energy by pressure, viscous stresses and Reynolds stresses, respectively. The second term (II) represents the dissipation of mean-flow kinetic energy due to the velocity gradients. This term is always negative and thus acts as a sink. The third term (III) accounts for the dissipation of the mean flow kinetic energy due to the Reynolds stresses. This term is usually negative, since the sign of Reynolds stresses is often opposite to the sign of the mean-flow velocity gradient.

\footnotetext{
${ }^{5}$ calculated by subtracting the loss within the $2 \mathrm{D}$ region from the $3 \mathrm{D}$ region
} 

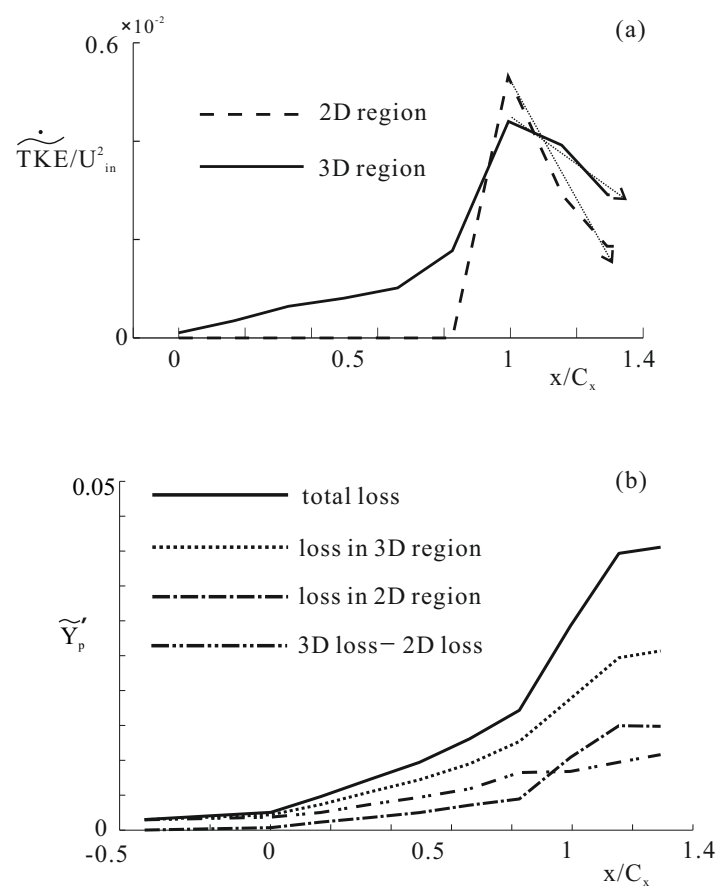

Figure 9: The development of (a) area-averaged turbulent kinetic energy and (b) mass-averaged total pressure loss coefficient in the blade passage.

Term III can also be described as turbulence production (but with an opposite sign) accounting for the transfer of energy from the mean flow into the turbulent fluctuations. In the subsequent analysis, it is assumed that the mean flow energy transferred to the turbulent scales dissipates eventually as heat without any backscattering.

The loss generation rate within the flow passage is governed by the terms II and III in equation 3, which are hereafter denoted as the mean flow Kinetic Energy Dissipation due to the velocity gradients $\left(K E D_{v i s}=2 \nu \bar{s}_{i j} \bar{s}_{i j}\right)$ and the turbulent Reynolds stresses $\left(K E D_{t u r}=-\overline{u_{i}^{\prime} u_{j}^{\prime}} \bar{s}_{i j}\right)$. Figure 10 shows the streamwise evolution of the area averaged $K E D_{v i s}$ and $K E D_{\text {tur }}$ within the blade passage. Three different zones are identified:

In zone $1\left(x / C_{x}<0\right)$, upstream of the blade leading edge, the 3D region alone contributes to the loss generation rate due to the wetted area at the endwall. In this zone, $K E D_{\text {vis }}$ and $K E D_{\text {tur }}$ contribute almost equally to the overall loss generation 


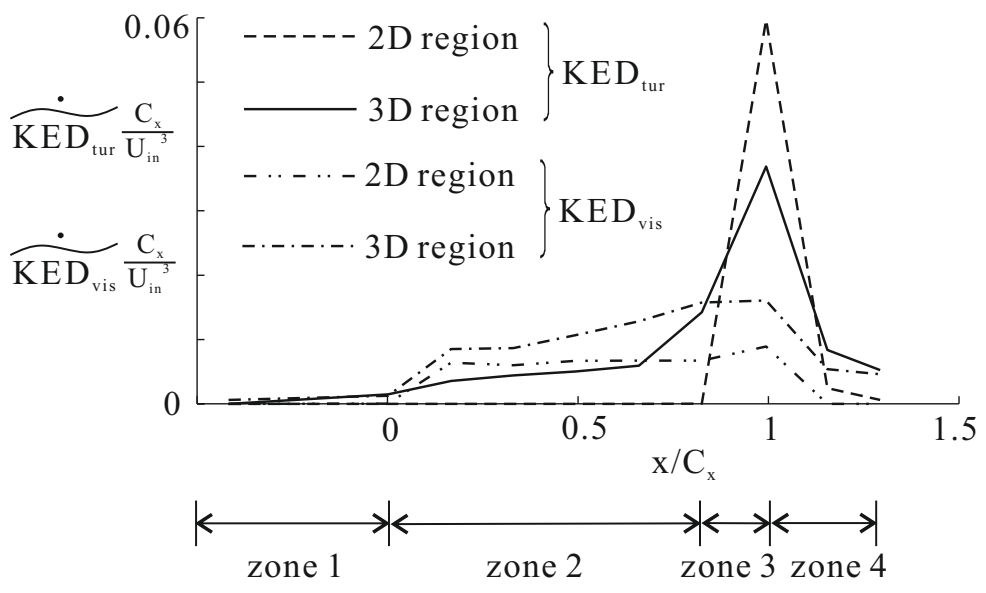

Figure 10: Mean flow energy dissipation due to velocity gradients $\left(K E D_{v i s}\right)$ and Reynolds stresses $\left(K E D_{\text {tur }}\right)$ along $x$-axial direction. rate in the $3 \mathrm{D}$ region.

Zone 2 corresponds to the region between the leading edge of the blade and upstream of the transition point $\left(x / C_{x}=0-0.84\right)$. In this zone, the loss generation within the $2 \mathrm{D}$ and $3 \mathrm{D}$ regions is largely due to $K E D_{\text {vis }}$. In the $2 \mathrm{D}$ region, the wetted area from the blade surface alone contributes to the loss generated by mean velocity gradients. However, both the blade surface and the endwall contribute to the loss generated by the mean velocity gradients in the $3 \mathrm{D}$ region. Thus, in zone $2, K E D_{\text {vis }}$ in the $3 \mathrm{D}$ region is larger than that of the $2 \mathrm{D}$ region. The boundary layers on the blade surface are largely laminar in the $2 \mathrm{D}$ region and thus will not contribute to the $K E D_{t u r}$. Contrarily, the disturbances due to $3 \mathrm{D}$ separation at the endwall increased $K E D_{t u r}$ in the $3 \mathrm{D}$ region, albeit their contribution being smaller than $K E D_{v i s}$.

In zone $3\left(0.84<x / C_{x}<1\right)$, where the boundary layer on the suction surface of the blade separates and transitions to turbulence, $K E D_{\text {tur }}$ increases sharply while the increase of $K E D_{v i s}$ is much more gradual. Additionally, $K E D_{t u r}$ increases faster in the $2 \mathrm{D}$ region relative to the $3 \mathrm{D}$ region. The reason for this is that the boundary layer separates and transitions to turbulence in the $2 \mathrm{D}$ region. However, in the $3 \mathrm{D}$ region, secondary flow suppresses part of the separation on the suction surface. Hence, $K E D_{t u r}$ in the $3 \mathrm{D}$ region is observed lower than in the $2 \mathrm{D}$ region at the trailing edge. 
In zone $4\left(x / C_{x}>1\right)$, downstream of the trailing edge, $K E D_{t u r}$ is almost twice as high as $K E D_{v i s}$ and thus it greatly contributes to the loss generation rate in both $2 \mathrm{D}$ and $3 \mathrm{D}$ regions. This is attributed to the violent production of turbulence from the separated shear layer transition in the $2 \mathrm{D}$ region and parts of 3D region. Notice that the loss generation rate due to turbulence $\left(K E D_{t u r}\right)$ in the $2 \mathrm{D}$ region decreases steeply between $x / C_{x}=1-1.16$. It almost drops to zero at $x / C_{x}=1.16$. However, there is still a noticeable level of $K E D_{t u r}$ in the $3 \mathrm{D}$ region. Recall from section 3.4 .2 that the turbulent kinetic energy in the $3 \mathrm{D}$ region decreased more gradually than that in the $2 \mathrm{D}$ (see figure 9).

The reason for this behaviour is demonstrated in figure 11, where the contours of $K E D_{\text {tur }}$ at $x / C_{x}=1.16$ and 1.3 are shown. Secondary flow velocity vectors and loss coefficient isolines are also superimposed. Three regions (A,B,C) with high $K E D_{\text {tur }}$ are identified in figure 11 (a). High $K E D_{\text {tur }}$ in: i) Region A corresponds to the dissipation of kinetic energy by the Reynolds stresses due to the convection of the low momentum fluid within the wake into the main stream, ii) region B corresponds to the convection of high velocity in the main stream into the wake, and iii) region $\mathrm{C}$ is caused by the shear layer on the suction side. As illustrated in the figure, the highest loss coefficient is associated with the counter vortex rather than the passage vortex. Also, the passage vortex loss core does not exactly collocate with the passage vortex core (indicated by secondary velocity vectors). These observations are consistent with the measurements of MacIsaac and Sjolander [13]. It is interesting to note that in figure 11 (b) the $K E D_{\text {tur }}$ term (which is identical to the turbulence production in TKE transport equation) in regions $\mathrm{A}$ and $\mathrm{B}$ are still relatively high while that in region $\mathrm{C}$ is not. It suggests that the passage vortex (in the $3 \mathrm{D}$ region) mixes out gradually when compared to the wake (in the $2 \mathrm{D}$ region) from the trailing edge. Thus, the contribution of $K E D_{\text {tur }}$ (and TKE) to the loss generation rate is reduced more slowly in the 3D region when compared to that in the $2 \mathrm{D}$ region.

\subsection{Effects of inflow condition}

Having analysed the baseline LBL case in the previous section, the effects of different inflow conditions (an incoming turbulent endwall boundary layer and wakes 

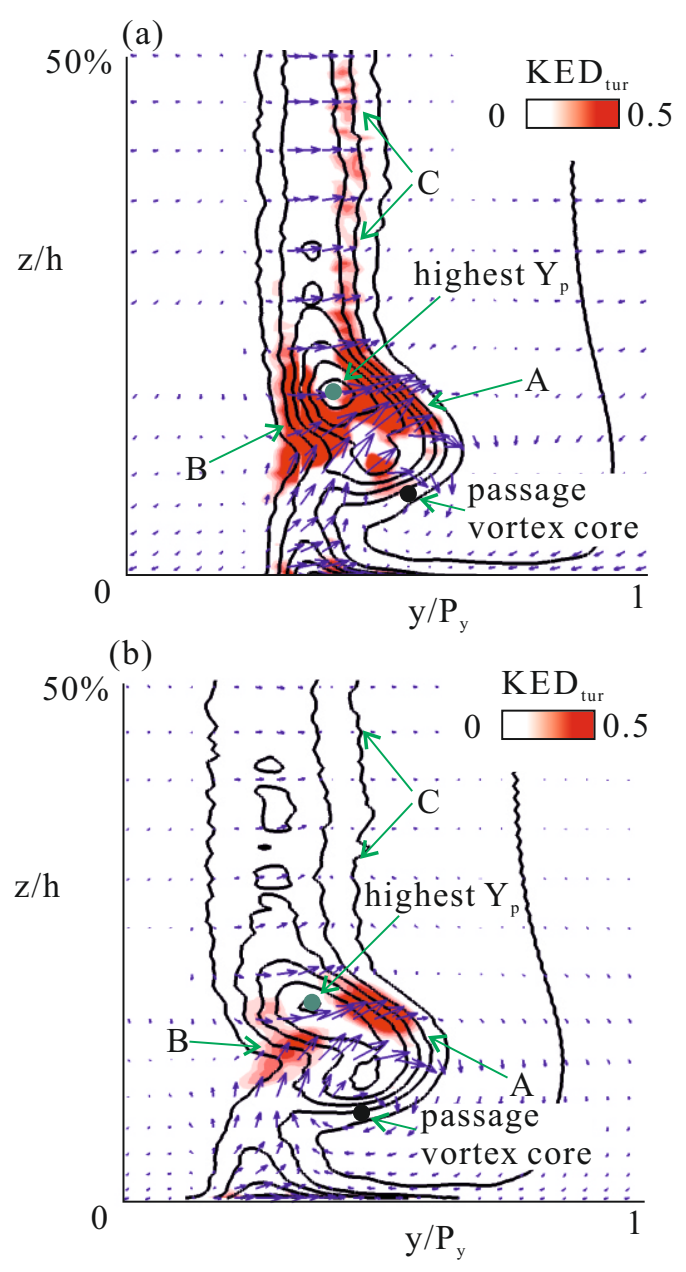

Figure 11: Local loss coefficient isolines with $K E D_{\text {tur }}$ contours and secondary velocity vectors superimposed at (a) $x / C_{x}=1.16$ and (b) $x / C_{x}=1.3$.

with secondary flows from the upstream rotor) on the loss generation are given in this section. The velocity profiles extracted upstream of the blade at $x / C_{x}=-0.1$ are shown in figure 12(a). Although it was ensured that the thickness of an incoming laminar boundary layer is same as that of an incoming tubulent boundary layer (see figure 3), the turbulent boundary layer grows thicker than the laminar boundary layer at $x / C_{x}=-0.1$. Note that a thicker boundary layer usually generates a higher endwall loss. 
Figure 12 (b) compares the axial variation of the mass averaged total pressure loss coefficient within the flow passage. When compared to the LBL case, the turbulent boundary layer has a negligible effect on the loss in the region of a favourable pressure gradient $\left(x / C_{x}<0.6\right)$. In this region, no interaction between the endwall flow and suction surface boundary layer is observed. As the endwall flow convects towards

305

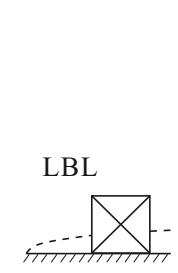

TBL
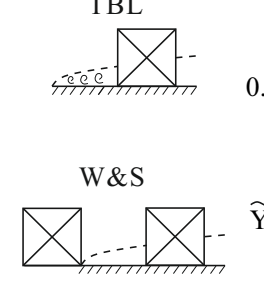

0.06

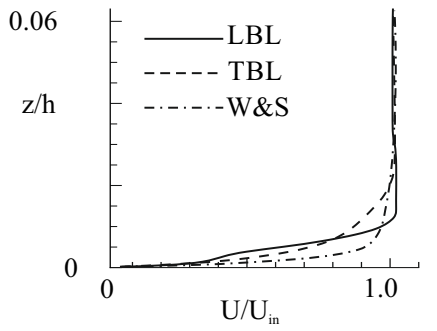

(a)

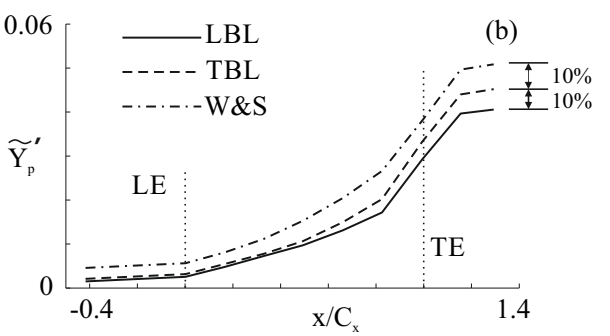

Figure 12: (a) Velocity profiles taken at $x / C x=-0.1$; (b) loss coefficient along $x$-axial direction.

The effect of inflow conditions on the dissipation of mean flow kinetic energy is examined. Following the analysis described in section 3.4 .3 , the axial evolution of the area averaged $K E D_{v i s}$ and $K E D_{t u r}$ within the blade passage are plotted in figure

13. Frame (a) shows the overall dissipation of mean kinetic energy. Prior to transition 

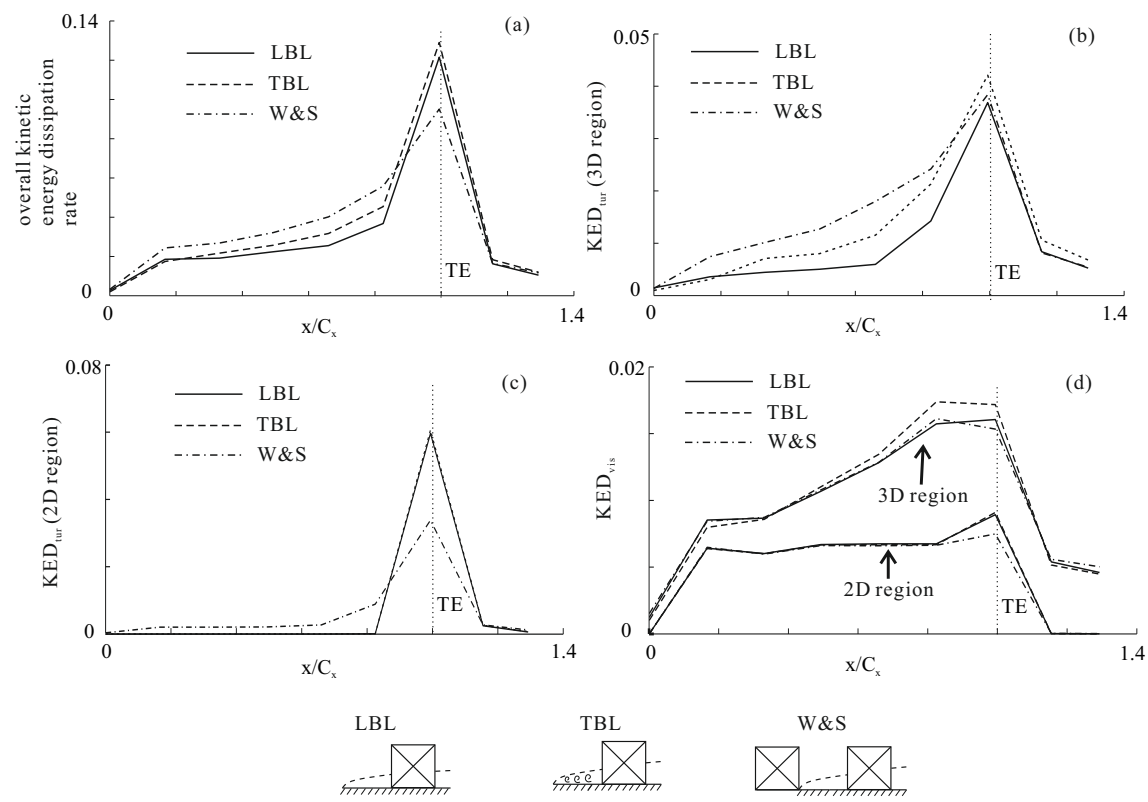

Figure 13: Loss generation rate within the passage for all the three cases: (a) overall loss generation rate; (b) loss generation rate due to $K E D_{\text {tur }}$ in the $3 \mathrm{D}$ and (c) 2D regions and (d) loss generation rate due to $K E D_{v i s}$.

$(x / C x<0.84)$, the presence of wakes with secondary flows contributed to the highest mean flow energy dissipation. However, notice that the trend has reversed downstream of the transition point. Frames (b) - (d) explain this behaviour considering the individual contributions of $K E D_{v i s}$ and $K E D_{t u r}$ in the 2D and $3 \mathrm{D}$ regions. $K E D_{v i s}$ (see frame (d)) is almost similar for all the cases with different inflow conditions; the contribution of the $3 \mathrm{D}$ region being larger than that of $2 \mathrm{D}$ due to a larger wetted area (from endwall and surfaces of the blade). However, there is a striking difference in the contribution of $K E D_{\text {tur }}$ with different inflow conditions (see frames (b,c)). In the $3 \mathrm{D}$ region, $K E D_{\text {tur }}$ increased progressively with increasing disturbances at the inflow; with the energy dissipation from W\&S being the largest. This is attributed to the higher turbulence intensities (and relatively larger velocity gradients) associated with the wakes and secondary flows from the upstream rotor convected into the blade passage. The incoming passage vortex mixes out gradually as it convects to the trailing edge, where the loss generation reduces to the similar level as the other two cases. At 
zero upstream of the transition point $(x / C x<0.84)$. However, the incoming wakes are effective in suppressing the separation bubble at the midspan thereby reducing the loss generation rate by $40 \%$ at the trailing edge.

\section{Conclusions}

Using a series of high-fidelity eddy resolving simulations, the current study attempts to provide an improved understanding of the endwall flows in high-lift low pressure turbines. Associated loss generation mechanisms were discussed in detail. The numerical framework has been validated by providing comparison against the measurements. The effect of three different inflow conditions have been studied. These include a laminar boundary layer, a turbulent boundary layer and wakes with secondary flow from an upstream blade row. The key results from the current study are:

- Eddy resolving simulations are helpful in accurately capturing the endwall flow features.

- When compared to LBL, the mass averaged total pressure loss $Y_{p}$ increased substantially in the presence of TBL and W\&S. This is attributed to the additional disturbances which enhanced mixing at the endwall, suction surface and within the passage.

- Upstream of the transition point $\left(x / C_{x}<0.84\right)$, the loss generation rate increased at both the endwall and at the midspan due to the incoming W\&S. After transition $\left(x / C_{x}>0.84\right)$, the presence of incoming wakes suppressed the 2Dseparation bubble at the midspan thereby decreasing the loss generation rate by almost $40 \%$.

- Downstream of the trailing edge $\left(x / C_{x}>1.0\right)$, the wake at the mid-span mixed out rapidly when compared to the passage vortex at the endwall. Thus the decrease in the loss generation rate at the endwall was much more gradual when compared to the steep decrease at the midspan. 


\title{
5. Acknowledgements
}

The authors would like to thank Rolls-Royce for providing the permission to publish this work. Acknowledgements are also due to the Cambridge Overseas Trust for 360 financially supporting this project. The authors would also like to acknowledge Dr.

Rob Watson, Dr. Ifthekar Naqavi and Mr. Ashly Scillitoe for their valuable discussions and supports.

\section{NOMENCLATURE}

\author{
$\delta^{*} \quad$ Boundary layer displacement thickness \\ $\theta \quad$ Boundary layer momentum thickness \\ $C_{x} \quad$ Axial chord \\ $C_{p} \quad$ Pressure coefficient $\left(P_{0 i n}-P\right) /\left(P_{0 i n}-P_{T E}\right)$ \\ $h \quad$ Span \\ $H \quad$ Shape factor \\ TKE Turbulent kinetic energy \\ $P \quad$ Local pressure \\ $P_{0} \quad$ Stagnation (total) pressure \\ $P_{y} \quad$ Pitch \\ $Q \quad Q$-criterion \\ Re Reynolds number based on $U_{T E}$ and $C$ \\ $U$ Velocity \\ $Y_{p} \quad$ Total pressure loss coefficient $\left(P_{0 i n}-P_{0}\right) /\left(P_{0 i n}-P_{T E}\right)$ \\ Subscripts \\ in or 1 Inlet \\ $T E$ or 2 Trailing edge or exit \\ Others \\ $\dot{\tilde{X}} \quad$ Area averaged quantity \\ $\tilde{X}^{\prime} \quad$ Mass averaged quantity
}


[1] M. M. Opoka, The effect of upstram and downstream bladerows on transition in low pressure turbines, Ph.D. thesis, University of Cambridge (2007).

口 [2] H. P. Hodson, R. J. Howell, The role of transition in high-lift low-pressure turbines for aeroengines, Progress in Aerospace Sciences 41 (6) (2005) 419-454. doi:10.1016/j.paerosci.2005.08.001. URL http://dx.doi.org/10.1016/j.paerosci.2005.08.001

口 [3] M. M. Opoka, H. P. Hodson, Experimental Investigation of Unsteady Transition Processes on High-Lift T106A Turbine Blades, Journal of Propulsion and Power 24 (3) (2008) 424-432. doi : 10.2514/1.31947. URL http://arc.aiaa.org/doi/abs/10.2514/1.31947

[4] H. Hodson, R. Howell, Unsteady flow: its role in the low pressure turbine, International Symposium Unsteady. URL http://www-g.eng.cam.ac.uk/whittle/publications/ hph/2000-ISUAAAT-LPReview.pdf

[5] J. Cui, V. N. Rao, P. Tucker, Numerical investigation of contrasting flow physics in different zones of a high-lift low-pressure turbine blade, Journal of Turbomachinery 138 (1) (2016) 011003.

[6] L. Langston, Secondary flows in axial turbinesła review, Annals of the New York Academy of Sciences 934 (1) (2001) 11-26.

[7] O. Sharma, T. Butler, Predictions of endwall losses and secondary flows in axial flow turbine cascades, Journal of Turbomachinery 109 (2) (1987) 229-236.

[8] H. Wang, S. J. Olson, R. J. Goldstein, E. R. Eckert, Flow visualization in a linear turbine cascade of high performance turbine blades, Journal of Turbomachinery 119 (1) (1997) 1-8.

[9] R. Goldstein, R. Spores, Turbulent transport on the endwall in the region between adjacent turbine blades, Journal of Heat Transfer 110 (4a) (1988) 862-869. 
[10] H. Hodson, R. Dominy, Three-dimensional flow in a low-pressure turbine cascade at its design condition, Journal of turbomachinery 109 (2) (1987) 177-185.

[11] J. Denton, G. Pullan, A numerical investigation into the sources of endwall loss in axial flow turbines, in: ASME Turbo Expo 2012: Turbine Technical Conference and Exposition, American Society of Mechanical Engineers, 2012, pp. 14171430.

[12] G. Ingram, D. Gregory-Smith, N. Harvey, Investigation of a novel secondary flow feature in a turbine cascade with end wall profiling, Journal of turbomachinery 127 (1) (2005) 209-214.

[13] G. MacIsaac, S. Sjolander, T. Praisner, Measurements of losses and reynolds stresses in the secondary flow downstream of a low-speed linear turbine cascade, Journal of Turbomachinery 134 (6) (2012) 061015.

[14] E. R. de la Blanco, H. Hodson, R. Vazquez, D. Torre, Influence of the state of the inlet endwall boundary layer on the interaction between pressure surface separation and endwall flows, Proceedings of the Institution of Mechanical Engineers, Part A: Journal of Power and Energy 217 (4) (2003) 433-441.

[15] A. Steurer, S. I. Benton, J. P. Bons, Effect of endwall boundary layer thickness on losses in a lpt cascade with unsteady wakes, in: ASME Turbo Expo 2014: Turbine Technical Conference and Exposition, American Society of Mechanical Engineers, 2014, pp. V02DT44A037-V02DT44A048.

[16] E. Blanco, Secondary flows in Low-Pressure Turbines, Ph.D. thesis, University of Cambridge (2004).

[17] T. S. Lund, X. Wu, K. D. Squires, Generation of Turbulent Inflow Data for Spatially-Developing Boundary Layer Simulations, Journal of Computational Physics 140 (2) (1998) 233-258. doi:10.1006/jcph.1998.5882.

\URL http://linkinghub.elsevier.com/retrieve/pii/ S002199919895882X 
[18] X. Wu, R. Jacobs, Simulation of boundary layer transition induced by periodically passing wakes Journal of Fluid Mechanics 398 (1999) 109-153. URL http://journals.cambridge.org/production/action/ cjoGetFulltext?fulltextid=15474

[19] L. Lapworth, S. Shahpar, Hydra-CFD: A framework for coldevelopment CFD development, in: International conference on scientific and engineering computi425 ation (IC-SEC), Singapore, July, 2004.

[20] R. Watson, Large eddy simulation of cutback trailing edges for film colling turbine blades, Ph.D. thesis, University of Cambridge (2013).

[21] S. E. Rogers, D. Kwak, C. Kiris, Steady and unsteady solutions of the incompressible navier-stokes equations, AIAA Journal 29 (4) (1991) 603-610. 